\title{
Relações hipotéticas entre os determinantes sociais da saúde que influenciam na obesidade em idosos
}

\author{
Hypothetical relationships between social determinants of health that influence on obesity \\ in the elderly
}

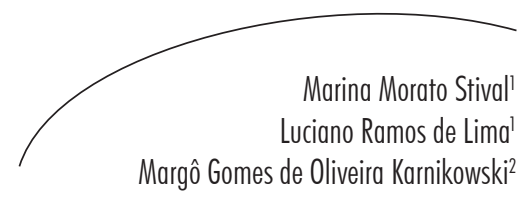

\section{Resumo}

Os elevados índices de prevalência de obesidade em idosos suscitam a necessidade de compreender os fatores envolvidos nesta desordem nutricional, por meio de métodos quantitativos que permitam uma análise relacional desses determinantes. O objetivo deste estudo foi propor um modelo hipotético que estabeleça as relações entre os determinantes sociais da saúde associados à obesidade em idosos. Para a construção do modelo hipotético, foram delineadas as variáveis latentes e observadas de acordo com a análise de 45 artigos nacionais e internacionais e em concordância com o referencial da Modelagem de Equações Estruturais. Foi construído um diagrama representativo para evidenciar as correlações entre os 11 determinantes sociais da saúde relacionados à obesidade no idoso: atividade física, tabagismo, etilismo, consumo alimentar, contato social, ocupação, renda, escolaridade, idade, sexo e estado civil. Espera-se que as relações hipotéticas estabelecidas no estudo contribuam para a compreensão das relações dos fatores que estão envolvidos nesse contexto visando ao desenvolvimento de estratégias para a saúde da pessoa idosa.

\section{Abstract}

The high prevalence rates of obesity in elderly raise the need to understand the factors involved in this nutritional disorder using quantitative methods that allow a relational analysis of these determinants. This study aimed to propose a hypothetical model to establish the relationship between the social determinants of health associated with obesity in elderly. For construction of the hypothetical model were delineated latent and observed variables according to the analysis of 45 national and international papers in reference of Structural Equation Modeling. It was-constructed a representative diagram to show the correlations between the eleven social of health related to obesity in the elderly: physical activity, smoking, alcohol consumption, dietary, social contact, occupation, income, education, age, sex and marital status. It is expected that the hypothetical relationships (established in this study contribute to the understanding of the relationships of factors involved in this context for the development of strategies for the health of the elderly.

\footnotetext{
Universidade de Brasília, Faculdade de Ceilândia, Curso de Enfermagem. Brasília, DF, Brasil.

2 Universidade de Brasília, Faculdade de Ceilândia, Curso de Farmácia. Brasília, DF, Brasil.
}

Palavras-chave: Obesidade. Idoso. Modelagem de Equações Estruturais.

Key words: Obesity. Elderly. Structural Equations Modeling. 


\section{INTRODUÇÃO}

A obesidade é uma desordem complexa e multifatorial que representa um complicador para o estado de saúde dos idosos e ocasiona ou potencializa as complicações advindas das doenças crônicas não transmissíveis associadas ao envelhecimento humano. Concomitantemente à transição demográfica, observa-se a transição epidemiológica e nutricional, com o aumento das doenças crônicas e elevação da prevalência de obesidade, sobretudo em idosos. ${ }^{1,2}$

O entendimento das relações existentes entre os determinantes sociais da saúde (DSS) envolvidos na obesidade contribuirá no sentido de direcionar a definição de estratégias para orientar a prevenção de agravos causados por esta desordem nutricional, bem como propiciará a organização da atenção à saúde e de programas de assistência aos idosos. ${ }^{3}$ É crescente o número de pesquisas que buscam estudar as associações entre a saúde dos idosos e os DSSs, mas a maioria dos estudos não estabelece as relações envolvidas entre tais determinantes. ${ }^{4}$ Nesse contexto, a discussão posta não se restringe a quantificar a obesidade em idosos com base em algum critério, mas buscar as relações que compõem o fenômeno. Compreender as relações dos fatores que influenciam direta ou indiretamente na obesidade poderá resultar em uma visão ampla e bem mais completa dessa relevante questão de saúde pública.

A obesidade é considerada objeto de pesquisas nacionais e internacionais com a utilização de diferentes métodos como estudos descritivos,${ }^{5}$ epidemiológicos ${ }^{1,6}$ e revisão da literatura., ${ }^{7,8}$ Entretanto, os métodos utilizados até o momento não conseguiram estabelecer as relações existentes entre os fatores envolvidos nesta desordem nutricional. ${ }^{3}$ Entre esses fatores, encontram-se aqueles denominados DSSs.

Para se estabelecer as relações entre os DSSs, tem-se utilizado análises estatísticas multivariadas, como a modelagem de equações estruturais (MEE), que se caracteriza por sua capacidade de especificar, estimar e testar relações hipotéticas entre um grupo de variáveis. ${ }^{9,10}$ Em linhas gerais, essa técnica de análise consiste em testar a plausibilidade de um modelo construído com base em uma teoria sobre o fenômeno estudado.

Sendo assim, o objetivo deste estudo foi investigar as relações entre os determinantes sociais da saúde associados à obesidade em idosos, e assim propor um modelo hipotético para testagem, por meio de modelagem de equações estruturais.

\section{METODOLOGIA}

Trata-se de um artigo de revisão integrativa que propõe a construção de um modelo hipotético de relações entre variáveis de acordo com o referencial da MEE. Para propor o modelo hipotético, é necessário delinear as variáveis que compõem o modelo. Estas podem ser classificadas como latentes (construtos) e observadas (indicadoras). As variáveis latentes (construtos latentes) não são diretamente mensuráveis, pois se referem a conceitos teóricos que não podem ser observados diretamente. Já as variáveis observadas são utilizadas para compor uma variável latente. ${ }^{11,12}$

Neste estudo, foram considerados os DSSs verificados em uma revisão integrativa e classificados em cada nível/conceito, que foram definidos de acordo com o Modelo de Dahlgren \& Whitehead. ${ }^{13}$ Realizou-se levantamento bibliográfico sobre as relações existentes na literatura entre os DSSs associados à obesidade em idosos no mês de janeiro de 2013. As buscas foram feitas nas bases de dados National Library of Medicine (PubMed) e Literatura LatinoAmericana em Ciências da Saúde (LILACS). Para a pesquisa de artigos, foram utilizados os descritores pelo DeCS - Descritores em Ciências da Saúde, em português e inglês, que abordassem os determinantes sociais da saúde: "obesidade", "idoso", "atividade motora", "estilo de vida sedentário", "tabagismo", "alcoolismo", "consumo de alimentos", "dieta", "apoio social", "ambiente", "sexo", "renda", "aposentadoria" e "escolaridade". A seleção do material bibliográfico 
ocorreu de acordo com os seguintes critérios de inclusão: artigos indexados no período de 2000 a 2012, com publicação completa em periódicos nacionais ou internacionais, nos idioma português e inglês, com abordagem quantitativa. Foram encontrados 745 artigos.

Estabeleceram-se os seguintes critérios de exclusão: artigo no formato editorial, comentário ou crítica, artigos de revisão de literatura e artigos que não tinham relação com a temática investigada. Após a leitura dos títulos e resumos, foram selecionados 45 artigos para a análise.

Para auxiliar na compreensão das relações estabelecidas, a partir das equações estruturais foi construído um modelo de representação gráfica com a utilização de um programa computacional Analysis of Moment Structures
(AMOS), um modo opcional do Software Package for the Social Sciences (SPSS), criado especificamente para realizar MEE. Trata-se de um software com uma interface gráfica (AMOS graphics) que inclui ferramentas de desenho de modelo, de análise de caminhos, além de calcular índices para verificar a adequação do modelo. ${ }^{14,15}$

\section{RESULTADOS}

No quadro 1, estão resumidas as referências bibliográficas que sustentaram as associações entre os DSSs a serem testadas sob a forma de um modelo de equações estruturais, de acordo com a análise dos 45 artigos. Todos os artigos analisados foram realizados com idosos e disponíveis na língua portuguesa ou inglesa.

Quadro 1. Referências bibliográficas selecionadas para sustentação das relações hipotéticas entre os determinantes sociais da saúde associados à obesidade em idosos. Brasília-DF, 2013.

\begin{tabular}{|c|c|}
\hline Correlação & Referências bibliográficas \\
\hline Sexo $\rightarrow$ Atividade física & $\begin{array}{l}\text { Siqueira et al. (2008); }{ }^{16} \text { Zaitune et al. (2007); }{ }^{17} \text { Zaitune et al. }(2010) ;{ }^{18} \\
\text { Paskulin \& Vianna (2007); }{ }^{19} \text { Azevedo et al. (2007); }{ }^{20} \text { Pitsavos et al. } \\
\text { (2005). }{ }^{21}\end{array}$ \\
\hline Renda $\rightarrow$ Atividade física & $\begin{array}{l}\text { Siqueira et al. (2008); }{ }^{16} \text { Zaitune et al. (2007); }{ }^{17} \text { Zaitune et al. (2010) }{ }^{18} \text { Lima- } \\
\text { Costa (2004). }{ }^{22}\end{array}$ \\
\hline Escolaridade $\rightarrow$ Atividade física & $\begin{array}{l}\text { Siqueira et al. (2008); }{ }^{16} \text { Zaitune et al. }(2010) ;{ }^{18} \text { Lima-Costa }(2004) ;{ }^{22} \text { Camões } \\
\text { \& Lopes (2008); }{ }^{23} \text { He \& Baker (2005); } ;{ }^{24} \text { Kaplan et al. (2001). }{ }^{25}\end{array}$ \\
\hline Tabagismo $\rightarrow$ Atividade física & $\begin{array}{l}\text { Zaitune et al. (2007); } ;^{17} \text { Zaitune et al. (2010); }{ }^{18} \text { Camões \& Lopes (2008); }{ }^{23} \\
\text { Kaplan et al. (2001); }{ }^{25} \text { Zaitune et al. (2012); }{ }^{26} \text { Monteiro et al. (2003); }{ }^{27} \text { Varo } \\
\text { et al. (2003). } .^{28}\end{array}$ \\
\hline Idade $\rightarrow$ Atividade física & $\begin{array}{l}\text { Zaitune et al. (2010); }{ }^{18} \text { Paskulin \& Vianna (2007); }{ }^{19} \text { Lima-Costa (2004); }{ }^{22} \\
\text { Camões \& Lopes (2008); }{ }^{23} \text { Cassou et al. (2008). }{ }^{29}\end{array}$ \\
\hline Ocupação $\rightarrow$ Atividade física & Zaitune et al. (2010); ${ }^{18}$ Camões \& Lopes (2008); ${ }^{23}$ Cassou et al. (2008). ${ }^{29}$ \\
\hline Etilismo $\rightarrow$ Atividade física & Zaitune et al. (2010); ${ }^{18}$ Mukamal et al. (2006). ${ }^{30}$ \\
\hline Contato social $\rightarrow$ Atividade física & Cassou et al. (2008). ${ }^{29}$ \\
\hline Sexo $\rightarrow$ Tabagismo & $\begin{array}{l}\text { Paskulin \& Vianna (2007); }{ }^{19} \text { Zaitune et al. (2012); }{ }^{26} \text { Peixoto et al. (2006); }{ }^{31} \\
\text { Peixoto et al. (2005);32 Marinho et al. (2008); }{ }^{33} \text { Senger et al. (2011; }{ }^{34} \text { Kim } \\
\text { \& Baik (2004;) })^{35} \text { Pereira et al. (2008). }{ }^{36}\end{array}$ \\
\hline Idade $\rightarrow$ Tabagismo & $\begin{array}{l}\text { Paskulin \& Vianna (2007); }{ }^{19} \text { Lima-Costa }(2004) ;{ }^{22} \text { Zaitune et al. }(2012) ;{ }^{26} \\
\text { Peixoto et al. (2005);32 Peixoto et al. (2006); }{ }^{31} \text { Drum et al. (2009); }{ }^{37} \text { Pereira } \\
\text { et al. (2008). }{ }^{36}\end{array}$ \\
\hline Renda $\rightarrow$ Tabagismo & $\begin{array}{l}\text { Lima-Costa (2004); }{ }^{22} \text { Zaitune et al. (2012); }{ }^{26} \text { Peixoto et al. (2006); }{ }^{31} \text { Peixoto } \\
\text { et al. (2005); } ;^{32} \text { Marinho et al. (2008); }{ }^{33} \text { Feliciano et al. (2004). }\end{array}$ \\
\hline
\end{tabular}




\begin{tabular}{|c|c|}
\hline Escolaridade $\rightarrow$ Tabagismo & $\begin{array}{l}\text { Lima-Costa (2004); }{ }^{22} \text { Peixoto et al. (2005); }{ }^{32} \text { Marinho et al. (2008); }{ }^{33} \text { Senger } \\
\text { et al. (2011); }{ }^{34} \text { Kim \& Baik (2004); }{ }^{35} \text { Costa et al. (2004). }{ }^{39}\end{array}$ \\
\hline Etilismo $\rightarrow$ Tabagismo & Marinho et al. (2008);33 Hirata et al. (2009);0 Paavola et al. (2004). ${ }^{41}$ \\
\hline Idade $\rightarrow$ Etilismo & $\begin{array}{l}\text { Zaitune et al. (2010); }{ }^{18} \text { Lima-Costa (2004); }{ }^{.2} \text { Pereira et al. }(2008) ;{ }^{36} \text { Drum et } \\
\text { al. (2009). }{ }^{37}\end{array}$ \\
\hline Sexo $\rightarrow$ Etilismo & $\begin{array}{l}\text { Zaitune et al. (2010); }{ }^{18} \text { Peixoto et al. (2006); }{ }^{31} \text { Senger et al. }(2011) ;{ }^{34} \text { Kim } \\
\text { \& Baik (2004); }{ }^{35} \text { Pereira et al. (2008); } ;{ }^{46} \text { Hirata et al. (2009) } ;{ }^{40} \text { Vieira et al. } \\
\text { (2010). }{ }^{42}\end{array}$ \\
\hline Escolaridade $\rightarrow$ Etilismo & $\begin{array}{l}\text { Zaitune et al. (2010); }{ }^{18} \text { Lima-Costa (2004); }{ }^{22} \text { Senger et al. (2011); }{ }^{34} \text { Kim \& } \\
\text { Baik (2004); }{ }^{35} \text { Costa et al. (2004); } ;{ }^{39} \text { Demaio et al. (2013). }{ }^{43}\end{array}$ \\
\hline Renda $\rightarrow$ Etilismo & Zaitune et al. (2010); ${ }^{18}$ Lima-Costa (2004); ${ }^{22}$ Feliciano et al. (2004). ${ }^{38}$ \\
\hline Ocupação $\rightarrow$ Etilismo & Zaitune et al. (2010) $;^{18}$ Pereira et al. (2008). ${ }^{36}$ \\
\hline Contato social $\rightarrow$ Etilismo & Senger et al. (2011). ${ }^{34}$ \\
\hline Sexo $\rightarrow$ Consumo alimentar & $\begin{array}{l}\text { Almeida et al. (2010); }{ }^{44} \text { Riedger \& Moghadasian (2008); }{ }^{45} \text { Appleton et al. } \\
\text { (2009); }{ }^{46} \text { Hall et al. (2009); }{ }^{47} \text { Salehi et al. (2010). }{ }^{48}\end{array}$ \\
\hline Idade $\rightarrow$ Consumo alimentar & Lima-Costa (2004); ${ }^{22}$ Riedger \& Moghadasian (2008); ${ }^{45}$ Hall et al. (2009). ${ }^{47}$ \\
\hline Renda $\rightarrow$ Consumo alimentar & Lima-Costa (2004); ${ }^{22}$ Riedger \& Moghadasian (2008); ${ }^{45}$ Hall et al. (2009). ${ }^{47}$ \\
\hline Tabagismo $\rightarrow$ Consumo alimentar & $\begin{array}{l}\text { Marinho et al. (2008);3 Senger et al. (2011); }{ }^{34} \text { Feliciano et al. (2004); }{ }^{38} \\
\text { Peltzer \& Phaswana-Mafuya (2012);9 Padrão et al. (2011). } .^{50}\end{array}$ \\
\hline Etilismo $\rightarrow$ Consumo alimentar & Senger et al. (2011); ${ }^{34}$ Feliciano et al.(2004); ${ }^{38}$ Padrão et al. (2011). ${ }^{50}$ \\
\hline $\begin{array}{l}\text { Escolaridade } \rightarrow \text { Consumo } \\
\text { alimentar }\end{array}$ & $\begin{array}{l}\text { Lima-Costa (2004); }{ }^{22} \text { Riedger \& Moghadasian (2008); }{ }^{45} \text { Peltzer \& } \\
\text { Phaswana-Mafuya (2012). }{ }^{49}\end{array}$ \\
\hline $\begin{array}{l}\text { Estado civil } \rightarrow \text { Consumo } \\
\text { alimentar }\end{array}$ & Riedger \& Moghadasian (2008); ${ }^{45}$ Li et al. (2012); ${ }^{51}$ Sahyoun et al. (2005). ${ }^{52}$ \\
\hline $\begin{array}{l}\text { Atividade física } \rightarrow \text { Consumo } \\
\text { alimentar }\end{array}$ & Li et al. (2012); ${ }^{51}$ Sahyoun et al. (2005). ${ }^{52}$ \\
\hline Idade $\rightarrow$ Contato Social & Paskulin \& Vianna (2007); $;^{19}$ Rosa (2005); $;{ }^{53}$ Rosa et al. $(2007) .{ }^{54}$ \\
\hline Renda $\rightarrow$ Contato Social & $\begin{array}{l}\text { Li et al. (2012); }{ }^{51} \text { Sahyoun et al. (2005); }{ }^{52} \text { Rosa }(2005) ;{ }^{53} \text { Rosa et al. (2007); } ;^{54} \\
\text { Pinto et a.l (2006). }\end{array}$ \\
\hline Sexo $\rightarrow$ Contato social & $\begin{array}{l}\text { Paskulin \& Vianna (2007); } ; \text { Pinto et al. (2006); }{ }^{55} \text { Rosa et al. (2007); }{ }^{54} \\
\text { Lima-Costa et al. (2000). }{ }^{56}\end{array}$ \\
\hline Estado civil $\rightarrow$ Contato social & Rosa et al. (2007); ${ }^{54}$ Pinto et al. (2006). ${ }^{55}$ \\
\hline Escolaridade $\rightarrow$ Contato social & Rosa et al. (2007); ${ }^{54}$ Pinto et al. (2006). ${ }^{55}$ \\
\hline Sexo $\rightarrow$ Escolaridade & $\begin{array}{l}\text { Paskulin \& Vianna (2007); }{ }^{19} \text { Lima-Costa et al. }(2000) ;{ }^{56} \text { Meireles et al. } \\
(2007) .{ }^{7}\end{array}$ \\
\hline Idade $\rightarrow$ Escolaridade & Paskulin \& Vianna (2007):;19 Lima-Costa (2004). ${ }^{22}$ \\
\hline Idade $\rightarrow$ Renda & Paskulin \& Vianna (2007); ${ }^{19}$ Campino \& Cirrylo (2003). ${ }^{58}$ \\
\hline Sexo $\rightarrow$ Ocupação & Paskulin \& Vianna (2007):;19 Lima-Costa (2004). ${ }^{22}$ \\
\hline Idade $\rightarrow$ Ocupação & Paskulin \& Vianna (2007); ${ }^{19}$ Giatti \& Barreto (2003). ${ }^{59}$ \\
\hline
\end{tabular}


O diagrama construído provê uma visão da maneira como se articulam os determinantes sociais da saúde associados à obesidade na população idosa. A representação gráfica foi construída em uma sequência lógica e temporal que acompanha as relações estabelecidas entre os DSSs analisados (figura 1).

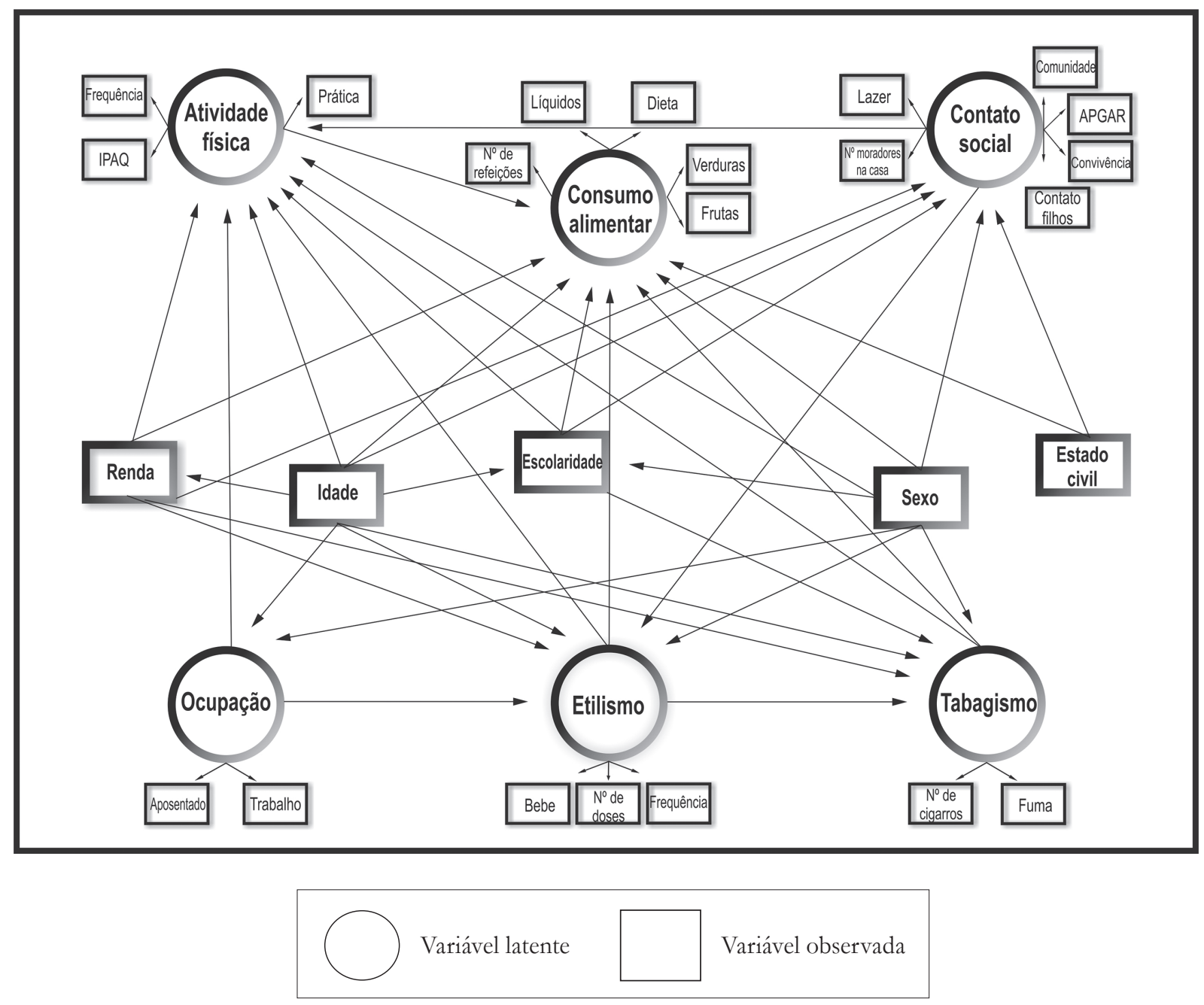

Figura 1. Diagrama representativo do modelo hipotético que estabelece as relações entre os determinantes sociais da saúde da obesidade em idosos. 
Após definidas as associações entre os DSSs relacionados à obesidade, estabeleceram-se as articulações por meio de construções de relações hipotéticas (hipóteses). Os DSSs associados à obesidade em idosos estão articulados entre si da seguinte maneira:

a) Idosos obesos do sexo feminino, com idade avançada, de baixa renda com baixa escolaridade, etilistas, tabagistas, aposentados e com pouco contato social são mais sedentários.

b) Idosos obesos do sexo masculino, com idade entre 60 e 69 anos, de baixa renda, com pouca escolaridade, etilistas e sedentários são mais tabagistas.

c) Idosos obesos do sexo masculino, com idade entre 60 e 69 anos, com mais anos de estudo, com maior renda, que trabalham e com maior contato social são mais etilistas.

d) Idosos obesos do sexo feminino, com idade avançada, maior renda, não fumante, não etilista, com mais anos de estudo, solteiros ou viúvos e que praticam atividade física têm melhor consumo alimentar.

e) Idosos obesos do sexo feminino, com idade entre 60 e 65 anos, com pouca renda, casados e com maior escolaridade têm mais contato social.

f) Idosos obesos do sexo feminino e com idade mais avançada têm menos anos de estudo.

g) Idosos obesos com idade avançada apresentam menores rendas.

h) Idosos obesos do sexo masculino apresentam mais atividade ocupacional. Aqueles com idade avançada apresentam maiores índices de aposentadoria.

\section{DISCUSSÃO}

Em uma análise sobre as metodologias aplicadas no estudo das relações entre os DSSs e a situação de saúde, os autores apontaram lacunas na compreensão da multidimensionalidade que envolvem esses fatores, evidenciadas pela atual produção científica, e fizeram recomendações para utilização de ferramentas e métodos mais eficazes. ${ }^{3}$

As pesquisas que relacionam variáveis não mensuráveis pelas metodologias estatísticas convencionais não permitem identificar e evidenciar as várias dimensões que influenciam e são influenciadas no contex to da obesidade, objeto desta pesquisa. Neste sentido, o presente estudo representa inovação ao utilizar a construção de um modelo que possibilita a aplicação da Modelagem de Equações Estruturais (MEE) para estabelecer as relações dos DSSs que se associam à obesidade em idosos. A MEE, que é uma técnica avançada, do ponto de vista da capacidade de articulação de variáveis sociais, permite calcular simultaneamente todas as relações entre os fatores associados a um fenômeno., ${ }^{910}$ Embora considerada relativamente recente e referenciada em publicações internacionais, a MEE ainda é pouco utilizada em publicações no país. ${ }^{11-60}$

Não foram encontrados estudos relatando associações entre os DSSs na população de idosos obesos, sendo que os resultados deste trabalho, que envolvem as relações hipotéticas, foram construídos com base em dados de pesquisas com a população idosa, não necessariamente com excesso de peso. No entanto, a metodologia de construção de relações hipotéticas vem sendo adotada em estudos de diferentes áreas ${ }^{10,61,62}$ para ser testada em um grupo específico por meio de MEE.

Para exemplificar, pesquisa desenvolvida em Salvador-BA, com o objetivo de construir um modelo hipotético das relações entre as variáveis sociais envolvidas no contexto da pobreza, concluiu que o modelo foi satisfatório, pois contribuiu para a percepção da multidimensionalidade desse fenômeno. ${ }^{63}$ Artigos de revisão foram publicados abordando a MEE, porém de maneira conceitual, teórica e prática. ${ }^{11,60,64}$ 
Essa técnica estatística é, portanto, utilizada quando se tem o objetivo de explicar as relações envolvidas em um fenômeno multidimensional. No estudo, a obesidade em idosos foi considerada pela sua multicomplexidade, uma vez que foram estabelecidas diversas hipóteses de relações entre os DSSs envolvidos nesta desordem nutricional. As equações foram construídas com base nessas relações, podendo ser testadas em um grupo de idosos com excesso de peso. Outros estudos também construíram equações hipotéticas com base em revisão da literatura, para serem testadas por meio de MEE. ${ }^{10,62,63}$

O modelo hipotético apresentado neste estudo, tanto na forma de equações matemáticas como no diagrama, retrata todas as relações possíveis entre as variáveis estabelecidas com base na literatura, ou seja, entre os DSSs associados à obesidade em idosos. No diagrama representativo, observam-se as correlações entre os 11 DSSs adotados como determinantes da obesidade no idoso: atividade física, tabagismo, etilismo, consumo alimentar, contato social, ocupação, renda, escolaridade, idade, sexo e estado civil.

Entre as variáveis em que foi encontrado maior número de relações, a atividade física e o consumo alimentar correlacionaram-se com oito DSSs diferentes, seguidos de etilismo, tabagismo e contato social (6 e 5 relações, respectivamente). Sexo, idade, renda e escolaridade se destacaram por se relacionarem com a maioria dos DSSs, mostrando-se como variáveis observadas que se relacionam com todos os níveis dos DSSs envolvidos na obesidade em idosos. Essas variáveis formam o contexto sociopolítico, que são fatores sociais determinantes das desigualdades na saúde, o que configura as diferentes oportunidades de saúde do idoso. ${ }^{4}$

O modelo hipotético eleborado no presente estudo possibilita a testagem utilizando a MEE para que tais relações sejam confirmadas ou rejeitas por meio do cálculo dos coeficientes de correlação., ${ }^{910}$ Não havendo confirmação satisfatória do modelo, tal processo deve prosseguircom os ajustes e adaptações necessárias, até que se resulte em uma representação aceitável das relações existentes entre os DSSs envolvidos no fenômeno da obesidade em idosos.

Acredita-se que, além da obesidade em idosos, outros fenômenos de magnitude na saúde possam ser beneficiados com as características e conceitos abordados neste estudo.

\section{CONSIDERAÇÕES FINAIS}

De maneira conclusiva, o estudo buscou mostrar que a multidimensionalidade inerente à obesidade em idosos requer métodos quantitativos específicos, que possibilitem uma abordagem de natureza complexa e relacional do fenômeno. Observaram-se correlações entre 11 determinantes sociais da saúde: atividade física, tabagismo, etilismo, consumo alimentar, contato social, ocupação, renda, escolaridade, idade, sexo e estado civil, tendo a atividade física e o consumo alimentar com maior número de relações.

Foi estabelecido um modelo hipotético que contribua para a compreensão das relações de todos os determinantes sociais da saúde envolvidos nesse contexto, visando ao desenvolvimento de estratégias para a saúde da pessoa idosa. Recomenda-se a realização de estudos que abordem a análise complexa da obesidade em diferentes grupos etários. A construção de relações hipotéticas por meio de modelagem de equações estruturais pode servir de base também para estudos futuros com outros objetos de interesse, para melhor compreensão de suas nuances relacionais. 


\section{REFERÊNCIAS}

1. Boscatto EC, Duarte MF, Coqueiro RS, Barbosa AR. Nutritional status in the oldest elderly and associated factors. Rev Assoc Med Bras [Internet]. 2013 [acesso em 05 out 2013];59(1):40-7. Disponível em: www. scielo.br/pdf/ramb/v59n1/v59n1a10.pdf

2. Newman AM. Obesity in older adults. The Online J Issues Nurs 2009;14(1):1-8.

3. Pellegrini A Filho. Public policy and the social determinants of health: the challenge of the production and use of scientific evidence. Cad Saúde Pública 2011;27(2):135-40.

4. Gieb LTC. Determinantes sociais da saúde do idoso. Ciênc Saúde Coletiva 2012;17(1):123-33.

5. Carvalho JA, Carvalho AP, Alves FA. Perfil Nutricional Associado ao Índice de Obesidade de Idosos do Centro de Saúde Sebastião Pinheiro Bastos, AAP-VR, Volta Redonda - RJ. Rev Praxis 2009;1(1):43-50.

6. Silva VS, Souza I, Petroski EL, Silva DAS Prevalência e fatores associados ao excesso de peso em idosos brasileiros. Rev Bras Ativ Física Saúde 2011;16(4):289-94.

7. Marques APO, Arruda IKG, Leal MCC, Santo ACGE. Envelhecimento, obesidade e consumo alimentar em idosos. Rev Bras Geriatr Gerontol 2007;10(2):231-42.

8. Wang Y, Beydoun MA. The obesity epidemic in the United States - gender, age, socioeconomic, racial/ ethnic, and geographic characteristics: a systematic review and meta-regression analysis. Epidemiol Rev 2007;29:6-28.

9. Kaplan D. Structural Equation Modeling: foundations and extensions. Thousand Oaks: Sage Publications; 2000.

10. Silva JSF. Modelagem de equações estruturais: apresentação de uma metodologia [dissertação]. Porto Alegre: Universidade Federal do Rio Grande do Sul, Faculdade de Engenharia de Produção; 2006.

11. Amorim LDAF, Fiaccone RL, Santos CAST, Santos TN, Moraes LTLP, Oliveira NF, et al. Structural equation modeling in epidemiology. Cad Saúde Pública 2010;26(12):2251-62.

12. Santos RB. Modelos de equações estruturais [dissertação]. Porto Alegre: Universidade Federal do Rio Grande do Sul, Instituto de Matemática; 2002.

13. Dahlgren G, Whitehead M. Policies and strategies to promote social equity in health. Stockholm: Institute for Future Studies; 1991.
14. Byrne B M. Structural equation modeling with AMOS: basic concepts, applications, and programming. Mahwah: Lawrence Erlbaum Associates; 2001.

15. Lemke C. Modelos de equações estruturais com ênfase em análise fatorial confirmatória no software AMOS [dissertação]. Porto Alegre: Universidade Federal do Rio Grande do Sul, Instituto de Matemática; 2005

16. Siqueira FV, Facchinni LA, Piccini RX, Tomasi E, Thumé E, Silveira DS, et al. Atividade física em adultos e idosos residentes em áreas de abrangência de unidades básicas de saúde de municípios das regiões Sul e Nordeste do Brasil. Cad Saúde Pública 2008;24(1):39-54.

17. Zaitune MPA, Barros MBA, Cesar CLG, Carandina L, Goldbaum M. Fatores associados ao sedentarismo no lazer em idosos, Campinas, São Paulo, Brasil. Cad Saúde Pública 2007;23(6):1329-38.

18. Zaitune MPA, Barros MBA, Cesar CLG, Carandina L, Goldbaum M, Alves MCGPl. Fatores associados à prática de atividade física global e de lazer em idosos: Inquérito de Saúde no Estado de São Paulo (ISA-SP), Brasil. Cad Saúde Pública 2010;26(8):1606-18.

19. Paskulin LMG, Vianna LAC. Perfil sociodemográfico e condições de saúde autoreferidas de idosos de Porto Alegre. Rev Saúde Pública 2007;41(5):757-68.

20. Azevedo MR, Araujo CLP, Reichert FF, Siqueira FV, Silva MC, Hallal PC. Gender differences in leisure-time physical activity. Int J Public Health 2007;52(1):8-15.

21. Pitsavos C, Panagiotakos DB, Lentzas Y, Stefanadis C. Epidemiology of leisure-time physical activity in socio-demographic, lifestyle and psychological characteristics of men and women in Greece: the ATTICA study. BMC Public Health 2005;5:30-7.

22. Lima-Costa MF. A escolaridade afeta, igualmente, comportamentos prejudiciais a saúde de idosos e adultos mais jovens?: Inquérito de Saúde da Região Metropolitana de Belo Horizonte, Minas Gerais, Brasil. Epidemiol Serv Saúde 2004;13(4):201-8.

23. Camões M, Lopes C. Fatores associados à atividade física na população portuguesa. Rev Saúde Pública 2008;42(2):208-16.

24. He XZ, Baker DW. Differences in Leisure-time, household, and work-related physical activity by race, ethnicity, and education. J Gen Intern Med 2005;20(3):259-66. 
25. Kaplan MS, Newsom JT, McFarland BH, Lu L. Demographic and psychosocial correlates of physical activity in late life. Am J Prev Med 2001;21(4):306-12.

26. Zaitune MPA, Barros MBA, Lima MG, Cesar CLG, Carandina L, Goldbaum M, et al. Fatores associados ao tabagismo em idosos: Inquérito de Saúde no Estado de São Paulo (ISA-SP). Cad Saúde Pública 2012;28(3):583-95.

27. Monteiro CA, Conde WL, Matsudo SM, Matsudo VR, Bonsenor IM, Lotufo PA. A descriptive epidemiology of leisure-time physical activity in Brazil, 1996-1997. Rev Panam Salud Pública 2003;14(4):246-54.

28. Varo JJ, Martinez-Gonzalez MA, Irala-Estevez J, Kearney J, Gibney M, Martinez JA. Distribution and determinants of sedentary lifestyles in the European Union. Int J Epidemiol 2003;32(1):138-46.

29. Cassou ACN, Fermino RC, Santos MS, RodriguezAnez CR, Reis RS. Barreiras para a atividade física em idosos: uma análise de grupos focais. Rev Educ Fís 2008;19(3):353-60.

30. Mukamal KJ, Ding EL, Djousse L. Alcohol consumption, physical activity, and chronic disease risk factors: a population-based cross-sectional survey. BMC Public Health 2006;6:111-8.

31. Peixoto SV, Firmo JOA, Lima-Costa MF. Condições de saúde e tabagismo entre idosos residentes em duas comunidades brasileiras (Projetos Bambuí e Belo Horizonte). Cad Saúde Pública 2006;22(9):1925-34.

32. Peixoto SV, Firmo JO, Lima-Costa MF. Factors associated to smoking habit among older adults (The Bambuí Health and Aging Study). Rev Saúde Pública 2005;39(5):745-53.

33. Marinho V, Blay SL, Andreoli SB, Gastal F. A prevalence study of current tobacco smoking in later life community and its association with sociodemographic factors, physical health and mental health status. Soc Psychiatr Psychiatr Epidemiol 2008;43(6):490-7.

34. Senger AEV, Ely LS, Gandolfi T, Schneider RH, Gomes I, Carli G. Alcoolismo e tabagismo em idosos: relação com ingestão alimentar e aspectos socioeconômicos. Rev Bras Geriatr Gerontol 2011;14(4):713-19.

35. Kim O, Baik S. Alcohol consumption, cigarette smoking, and subjective health in Korean elderly men. Addict Behav 2004;29(8):1595-603.

36. Pereira JC, Barreto SM, Passos VMA. O perfil de saúde cardiovascular dos idosos brasileiros precisa melhorar: estudo de base populacional. Arq Bras Cardiol 2008;91(1):1-10.
37. Drum ML, Shiovitz-Ezra S, Gaumer E, Lindau ST. Assessment of smoking behaviors and alcohol use in the national social life, health, and aging project. J Gerontol Ser B, Psychol Sci Soc Sci 2009;64(Suppl 1):119-30.

38. Feliciano AB, Moraes AS, Freitas ICM. O perfil do idoso de baixa renda no Município de São Carlos: um estudo epidemiológico. Cad Saúde Pública 2004;20(6):1575-85.

39. Costa JSD, Silveira MFS, Gazalle FK, Oliveira SS, Hallal PC, Menezes AMB, et al. Consumo abusivo de álcool e fatores associados: estudo de base populacional. Rev Saúde Pública 2004;38(2):284-91.

40. Hirata ES, Nakano EY, Pinto JA Junior, Litvoc J, Bottino CMC. Prevalence and correlates of alcoholism in community-dwelling elderly living in São Paulo, Brazil. Int J Geriatr Psychiatry 2009;24(10):1045-53.

41. Paavola M, Vartiainen E, Haukkala A. Smoking, alcohol use, and physical activity: a 13-year longitudinal study ranging from adolescence into adulthood. J Adolesc Health 2004;35(3):238-44.

42. Vieira VL, Fava DBC, Komatsu TR, Andrade SC, Mancuso AM, Tanaka ACDA. Perfil nutricional de participante da campanha nacional de vacinação do idoso em um centro de saúde escola do município de São Paulo. Rev Bras Nutr Clín 2010;25(2):171-6.

43. Demaio AR, Dugee O, Courten M, Bygbjerg IC, Enkhtuya P, Meyrowitsch DW. Exploring knowledge, attitudes, and practices related to alcohol in Mongolia: a national population-based survey. BMC Public Health 2013;13:178-84.

44. Almeida IC, Guimarães GF, Rezende DC. Hábitos alimentares da população idosa: padrões de compra e consumo. Gest Contemp 2010;7(8):63-92.

45. Riedger ND, Moghadasian MH. Patterns of fruit and vegetable consumption and influence of sex, age and sociodemographic factors among Canadian elderly. J Am College Nutr 2008;27(2):306-13.

46. Appleton KM, McGill R, Woodside JV. Fruit and vegetable consumption in older individuals in Northern Ireland: levels and patterns. Br J Nutr 2009;102(7):949-53.

47. Hall JN, Moore S, Harper SB, Lynch JW. Global variability in fruit and vegetable consumption. Am Prev Med 2009;36(5):402-9.

48. Salehi L, Eftekhar H, Mohammad K, Tavafian SS, Jazayery A, Montazeri A. Consumption of fruit and vegetables among elderly people: a cross sectional study from Iran. Nutr J 2010;13(2):1-9. 
49. Peltzer K, Phaswana-Mafuya N. Fruit and vegetable intake and associated factors in older adults in South Africa Citation. Glob Health Action 2012;5:18668-76.

50. Padrão P, Silva-Matos C, Damasceno A, Lunet N. Association between tobacco consumption and alcohol, vegetable and fruit intake across urban and rural areas in Mozambique. J Epidemiol Community Health 2011;65(5):445-53

51. Li Y, Li D, Ma CY, Liu CY, Huni-Ding, Wen ZM, et al. Consumption and factors influencing consumption of fruit and vegetables among elderly Chinese people. Nutrition 2012;28(5):504-8.

52. Sahyoun NR, Zhang XL, Serdula MK. Barriers to the consumption of fruits and vegetables among older adults. J Nutr Elder 2005;24(4):5-21.

53. Rosa TEC. Determinantes do estado nutricional de idosos do município de São Paulo: fatores socioeconômicos, redes de apoio social e estilo de vida [tese]. São Paulo: Faculdade de Saúde Pública; 2005.

54. Rosa TEC, Benicio MHD, Alves MCGP, Lebrao ML. Aspectos estruturais e funcionais do apoio social de idosos do Município de São Paulo, Brasil. Cad Saúde Pública 2007;23(12):2982-92.

55. Pinto JLG, Garcia ACO, Bocchi SCM, Carvalhaes MABL Características do apoio social oferecido a idosos de área rural assistida pelo PSF. Ciênc Saúde Coletiva 2006;11(3):753-64.

56. Lima-Costa MF, Uchoa E, Guerra HL, Firmo JOA, Vidigal PG, Barreto SM. The Bambuí Health and Ageing Study (BHAS): methodological approach and preliminary results of a population-based cohort study of the elderly in Brazil. Rev Saúde Pública 2000;34(2):126-35.
57. Meireles VC, Matsuda LM, Coimbra JAH, Mathias TAF. Características dos Idosos em Área de Abrangência do Programa Saúde da Família na Região Noroeste do Paraná: contribuições para a gestão do cuidado em enfermagem. Saúde Soc 2007;16(1):69-80.

58. Campino ACC, Cyrillo DC. Situação de ocupação e renda. In: Lebrão Ml, Duarte YAO. SABE - saúde, Bem-estar e Envelhecimento. O projeto SABE no município de São Paulo: uma abordagem inicial. Brasília, DF:Organização Pan-Americana de Saúde; 2003. p.241-54.

59. Giatti L, Barreto SM. Saúde, trabalho e envelhecimento no Brasil. Cad Saúde Pública 2003;19(3):759-71.

60. Codes AL. Modelagem de equações estruturais: um método para análise de fenômenos complexos. Caderno CRH 2005;18(45):471-84.

61. Olson K, Hayduk L, Cree M, Cui Y, Quan H, Hanson J, et al. The changing causal foundations of cancerrelated symptom clustering during the final month of palliative care: a longitudinal study. BMC Med Res Methodol 2008;8:30-6.

62. Shah S, Novak S, Stapleton LM. Evaluation and comparison of models of metabolic syndrome using confirmatory factor analysis. Eur J Epidemiol 2006;21(5):343-9.

63. Lima ALMC. Modelagem de Equações Estruturais: uma contribuição metodológica para o estudo da pobreza [tese]. Salvador: Universidade Federal da Bahia; 2005.

64. Pilati R, Laros JA. Modelos de equações estruturais em psicologia. Psicol Teor Pesqui 2007;23(2):205-16. 